\title{
In vitro activity of levofloxacin against lower respiratory tract pathogens
}

\begin{abstract}
Background: Considerable morbidity and mortality are associated with lower respiratory tract infections (LRTIs) that put a considerable strain on the health budget. Selection of appropriate antibiotics as empirical therapy maximizes positive patient outcomes, and that depends on regular surveillance of infective agents and their antibiograms, which vary according to the geographical areas.

Aim: The aim was to study the drug susceptibility pattern of the isolated pathogens of the respiratory tract infections.

Settings and Design: Retrospective study for a period of 1-year 3 months from January 2013 to March 2014 at a Tertiary Care Hospital.

Materials and Methods: Eleven hundred and eighty-four sputum samples from both outdoor and indoor patients with symptoms of LRTI were processed, and antibiotic sensitivity test was done to commonly used antibiotics. Descriptive statistics was used to analyze the data.

Results: Among 502 quality sputum samples, 312 (62.15\%) samples showed growth of pathogenic bacteria. The most common pathogens were Klebsiella spp. (38.14\%), Moraxella spp. (16.02\%), Streptococcus pneumoniae (14.10\%), Pseudomonas spp. (9.93\%), S. aureus (9.29\%). It was found that the overall susceptibility pattern was $<50 \%$ for amoxicillin, amoxicillin-clavulanic acid, cefuroxime, cotrimoxazole and erythromycin whereas for cefotaxime, cefixime, and cefoperazone-sulbactum it was $60.08 \%, 51.59 \%, 69.04 \%$, respectively. The susceptibility to ciprofloxacin, ofloxacin, and levofloxacin were $66.67 \%, 70.19 \%$ and $83.33 \%$, respectively.

Conclusion: Klebsiella spp. was the most common LRTI pathogen. There was limited activity of amoxicillin, amoxicillin-clavulanic acid, cefuroxime, cotrimoxazole and erythromycin for the treatment of LRTI whereas levofloxacin, (being an oral drug with good compliance) had good activity against respiratory pathogens and could be used for empiric treatment in LRTI.
\end{abstract}

\section{Key words:}

Antimicrobial susceptibility patterns, levofloxacin, lower respiratory tract infections

\section{Introduction}

Lower respiratory tract infections (LRTIs) include mainly chronic bronchitis and its acute exacerbation and pneumonia. Etiology of LRTIs depend on various demographic characteristics that include the place of study (rural/urban), age (young/middle/old) and other predisposing factors, including hospitalization. Nosocomial and community-acquired LRTIs have been on the rise as is the case with other debilitated conditions

\begin{tabular}{|l|c|}
\hline \multicolumn{2}{|c|}{ Access this article online } \\
\hline \multirow{2}{*}{$\begin{array}{l}\text { Website: } \\
\text { www.jbclinpharm.org }\end{array}$} & Quick Response Code \\
\hline DOI: & \\
10.4103/0976-0105.160749 & \\
&
\end{tabular}

that include compromised respiratory tract (asthma, chronic obstructive pulmonary disease [COPD]), diabetes, chronic kidney disease. ${ }^{[1-3]}$ Nosocomial LRTIs are mainly caused by Gram-negative organisms (Klebsiella spp., Escherichia coli, Pseudomonas, Acinetobacter spp.) whereas community-acquired LRTI are Streptococcus pneumoniae, Haemophilus influenzae, Moraxella catarrhalis, Staphylococcus aureus. Besides, Chlamydia and Mycoplasma pneumoniae also cause atypical pneumonias.
Soma Sarkar, Atreyi Chakraborty, Mallika Sengupta', Sougata Ghosh, Subhasish Mukhopadhyay, Manideepa SenGupta Department of Microbiology, Medical College, Kolkata,West Bengal, 'Department of Microbiology, CMC Vellore, Tamil Nadu, India
Address for correspondence: Dr. Soma Sarkar, Department of Microbiology Medical College, 88 College Street, Kolkata - 700 073, West Bengal, India. E-mail:drdssarkar@gmail.com


Antibiotics are often prescribed to treat LRTIs; however, these are not indicated in viral infections. It is important to use appropriate antibiotic based on the causative organism and the therapy changes with the emerging infections and the emerging resistance to conventional therapies. ${ }^{[4]}$

Among LRTI pathogens, production of $\beta$-lactamase among $H$. influenzae, $M$. catarrhalis and many Gram-negative bacilli have led to their development of resistance to first-line therapy.

Oral antibiotics are preferred by the clinicians because of good patient's compliance and because patients can be treated on an outpatient department (OPD) basis. Among oral antibiotics, quinolones are well absorbed, with moderate to excellent bioavailability. ${ }^{[5,6]}$ Serum drug levels achieved after oral administration are comparable to those with intravenous dosing, which allow an early transition from intravenous to oral therapy and a potential reduction of treatment cost. ${ }^{[7]}$

Since the introduction of nalidixic acid, the first quinolone, in 1962 structural modifications have resulted in second-, third-, and fourth-generation fluoroquinolones, which have improved coverage of Gram-positive organisms. ${ }^{[8]}$ As a general rule, Gram-negative bactericidal activity correlates with inhibition of DNA gyrase, and Gram-positive bactericidal activity corresponds with inhibition of DNA type IV topoisomerase. ${ }^{[8]}$

Thus, the aim of this study was to isolate the pathogens causing LRTIs and to compare the in vitro efficacy of levofloxacin among common fluroquinolones and among other commonly used first line antibiotics by disc diffusion method as well as by their minimum inhibitory concentrations (MICs) against major respiratory pathogens.

\section{Materials and Methods}

After getting the institutional ethical clearance, this study was conducted in the Department of Microbiology, Medical College, Kolkata from January 2013 to March 2014. Five hundred and two quality sputum samples were selected from 1184 patients having LRTI who either came to OPD or were admitted in the hospital. Specimens containing more than 25 polymorphonuclear leukocytes and $<10$ epithelial cells per low-power field were included in the study. ${ }^{[9]}$

Patients suffering from upper respiratory infections were not included in this study. Sputum samples were inoculated onto 5\% sheep blood agar, chocolate agar and MacConkey's agar media and incubated at $37^{\circ} \mathrm{C}$ overnight both aerobically as well as in a candle jar for isolation of Pneumococcus and Haemophilus spp. Further identifications were done according to standard microbiology criteria using conventional methods. The antimicrobial susceptibility of various microorganisms were tested against amoxicillin (10 $\mu \mathrm{g})$, amoxicillin-clavulanic acid (20/10 $\mu \mathrm{g})$, cefuroxime (30 $\mu \mathrm{g}$ ), cefotaxime (30 $\mu \mathrm{g}$ ), cefoperazone-sulbactum cefixime $(5 \mu \mathrm{g})$, erythromycin $(15 \mu \mathrm{g})$, azithromycin $(15 \mu \mathrm{g})$, ciprofloxacin $(5 \mu \mathrm{g})$, ofloxacin $(5 \mu \mathrm{g})$, levofloxacin $(5 \mu \mathrm{g})$, doxycycline $(30 \mu \mathrm{g})$, cotrimoxazole $(1.25 / 23.75 \mu \mathrm{g})$ using
Kirby-Bauer disk diffusion method. E. coli ATCC 25922, Pseudomonas aeruginosa ATCC 27853 and $S$. aureus ATCC 25923 were used as controls. Extended-spectrum beta-lactamase (ESBL) production were detected by disc synergy method.

MIC values of levofloxacin, ciprofloxacin and ofloxacin were determined by the E-test (Hi-Media Laboratories Pvt. Ltd.) method on Mueller-Hinton agar (MHA), on MHA with 5\% sheep blood for $S$. pneumoniae, and on Haemophilus test medium for $H$. influenzae strains.

\section{Results}

Patient's profile of lower respiratory tract infection Table 1 shows age-wise distribution of patients with LRTI. The majority of patients with LRTI were male 211 (67.6\%) with male: Female ratio 2.09:1.

\section{Sputum isolates}

Among 502 quality sputum samples, 312 (62.15\%) samples showed growth of pathogenic bacteria. Two hundred and seventy-seven isolates were from in-patient department and 35 isolates werefrom OPD. Figure 1 shows ward-wise distribution of the pathogens. The predominant bacterial species in LRTI were Klebsiella spp. (119 isolates), Moraxella spp. (50 isolates), Pneumococcus spp. (44 isolates), P. aeruginosa (31 isolates), $S$. aureus (29 isolates), E. coli spp. (14 isolates), Acinetobacter spp. (12 isolates), Haemophilus spp. (7 isolates), Citrobacter spp. (6 isolates). Among a total 312 isolates 201 (64.42\%) causing community-acquired pneumonia (CAP) and 111 (35.58\%) causing hospital-acquired pneumonia (HAP) [Table 2].

\begin{tabular}{lcc}
\hline $\begin{array}{l}\text { Table 1: Age wise distribution of patients with LRTI } \\
\text { Age-group } \\
\text { (years) }\end{array}$ & $\begin{array}{c}\text { Number of patients } \\
\text { having LRTI }\end{array}$ & $\begin{array}{c}\text { Percentage of patients } \\
\text { having LRTI (\%) }\end{array}$ \\
$<15$ & 10 & 3.20 \\
$15-30$ & 58 & 18.60 \\
$30-45$ & 73 & 23.40 \\
$45-60$ & 93 & 29.80 \\
$>60$ & 78 & 25 \\
\hline
\end{tabular}

LRTI: Lower respiratory tract infections

\section{Table 2: LRTI pathogens causing CAP and HAP}

\begin{tabular}{lcc} 
Number of isolates & CAP (\%) & HAP (\%) \\
\hline Klebsiella spp. (119) & $81(68.06)$ & $38(31.94)$ \\
Moraxella spp. (50) & $44(88)$ & $6(12)$ \\
Pneumococcus (44) & $38(83.36)$ & $6(13.64)$ \\
Pseudomonas spp. (31) & $9(29.03)$ & $22(70.97)$ \\
Staphylococcus aureus (29) & $16(55.17)$ & $13(44.83)$ \\
Escherichia coli (14) & $2(14.28)$ & $12(85.72)$ \\
Acinetobacter spp. (12) & $2(16.66)$ & $10(83.34)$ \\
Hemophilus influenzae (7) & $7(100)$ & $0(0)$ \\
Citrobacter spp. (6) & $2(33.33)$ & $4(66.67)$ \\
Total isolates $=312$ & $201(64.42)$ & $111(35.58)$ \\
\hline
\end{tabular}

CAP: Community acquired pneumonia, HAP: Hospital acquired pneumonia, LRTI: Lower respiratory tract infections 


\begin{tabular}{|c|c|c|c|c|c|c|c|c|c|c|c|c|}
\hline & \multicolumn{12}{|c|}{ Number/percentage of resistance of different first line antibiotics against LRTI pathogens } \\
\hline & AMX & AMC & CXM & CTX & CFS & CFX & $\mathrm{E} / \mathrm{Az}$ & CIP & OF & LE & DO & СOT \\
\hline Klebsi & $108 / 90.7$ & $98 / 82.3$ & $99 / 83.2$ & $66 / 55.5$ & $31 / 26$ & $84 / 70.6$ & $102 / 85.7$ & $46 / 38.7$ & $42 / 35.3$ & $24 / 20.2$ & $72 / 60.5$ & $101 / 84$ \\
\hline & 20 & $4 / 8$ & $9 / 18$ & 1 & 9/18 & $6 / 12$ & 8/ & $6 / 12$ & $6 / 12$ & $4 / 8$ & $8 / 16$ & $36 / 72$ \\
\hline Pneum & $10 / 22.7$ & $6 / 13.6$ & $4 / 9$ & $2 / 4.5$ & . & $5 / 11.4$ & $6 / 13.6$ & $16 / 36.4$ & $11 / 25$ & 6/13.6 & $4 / 9.0$ & $28 / 63.6$ \\
\hline Pseu & 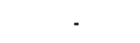 & - & . & $20 / 64.5$ & $16 / 51.6$ & - & - & $8 / 25.8$ & $10 / 32.3$ & $5 / 16.1$ & - & - \\
\hline reus (29) & $24 / 82.8$ & $2 / 75.9$ & $18 / 62$ & . & . & . & $21 / 72.4$ & $11 / 37.9$ & $9 / 31$ & $2 / 6.9$ & $9 / 31$ & $23 / 79.3$ \\
\hline Escher & $13 / 92.9$ & $11 / 78.6$ & $12 / 85.7$ & $10 / 71.4$ & $9 / 64.3$ & $12 / 85.7$ & - & 10/71.4 & $9 / 64.3$ & $6 / 42.9$ & $8 / 57.1$ & $14 / 100$ \\
\hline Acinetobacter spp. (12) & $11 / 91.7$ & $10 / 83.3$ & $11 / 91.7$ & $7 / 58.3$ & $6 / 50$ & $10 / 83.3$ & - & $4 / 33.3$ & $4 / 33.3$ & $3 / 25$ & $8 / 66.7$ & $11 / 91.7$ \\
\hline Haem & & & & مו & 0 & 0 & $1 / 14.2$ & & 0 & 0 & & $3 / 42.9$ \\
\hline Citrobacter spp. (6) & $5 / 83.3$ & $4 / 66.6$ & $5 / 83.3$ & $4 / 66.6$ & $3 / 50$ & $5 / 83.3$ & 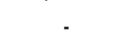 & $2 / 33.3$ & $2 / 33.3$ & $2 / 33.3$ & $3 / 50$ & $4 / 66.6$ \\
\hline
\end{tabular}

AMX: Amoxicillin $(10 \mu \mathrm{g})$, AMC: Amoxicillin-clav $(20 / 10 \mu \mathrm{g})$, CXM: Cefuroxime $(30 \mu \mathrm{g})$, CTX: Cefotaxime $(30 \mu \mathrm{g})$, CFS: Cefoperazone-sulbactum CFM: Cefixime $(5 \mu \mathrm{g})$, E: Erythromycin $(15 \mu \mathrm{g})$, Az: Azithromycin $(15 \mu \mathrm{g})$, CIP: Ciprofloxacin $(5 \mu \mathrm{g})$, OF: Ofloxacin $(5 \mu \mathrm{g}), \mathrm{LE}$ : Levofloxacin $(5 \mu \mathrm{g})$, DO: Doxycycline $(30 \mu \mathrm{g})$, COT: Cotrimoxazole $(1.25 / 23.75 \mu \mathrm{g})$, LRTI: Lower respiratory tract infections

\section{Table 4: The overall susceptibility pattern of different first line antibiotics against common LRTI pathogens}

\begin{tabular}{lccc} 
Antibiotics & $\begin{array}{c}\text { Percentage } \\
\text { of sensitive }\end{array}$ & $\begin{array}{c}\text { Percentage of } \\
\text { intermediate } \\
\text { sensitive }\end{array}$ & $\begin{array}{c}\text { Percentage } \\
\text { of resistant }\end{array}$ \\
\hline Amoxicillin & 34.88 & 0 & 65.12 \\
Amoxicillin-clav & 44.49 & 0 & 55.51 \\
Cefuroxime & 39.26 & 4.16 & 56.58 \\
Cefotaxime & 56.3 & 3.78 & 39.92 \\
Cefoperazone sulbactum & 56.08 & 12.96 & 30.96 \\
Cefixime & 47.11 & 4.48 & 48.41 \\
Erythromycin & 44.58 & 0 & 55.42 \\
Ciprofloxacin & 60.36 & 6.31 & 33.33 \\
Ofloxacin & 63.46 & 6.73 & 29.81 \\
Levofloxacin & 72.3 & 11.03 & 16.67 \\
Doxycycline & 60.14 & 0 & 39.86 \\
Cotrimoxazole & 21.71 & 0 & 78.29 \\
\hline
\end{tabular}

LRTI: Lower respiratory tract infections

\section{Antibiotic susceptibility testing}

Klebsiella species were least resistant to cephoperazone sulbactum and fluoroquinolones, and highly resistant to amoxicillin (90.7\%). 31 (26.05\%) Klebsiella isolates were ESBL producer. Moraxella species showed good susceptibility to amoxicillin-clav, fluroquinolones, cephalosporins and erythromycin. The antibiotic resistance patterns of the remaining organisms are shown in Table 3.

The overall susceptibility pattern of antibiotics against LRTI pathogens was the highest to levofloxacin (83.33\%), ofloxacin (70.19\%) and ciprofloxacin (66.67\%) and lowest to co-trimoxazole (21.21\%) followed by amoxicillin (34.88\%) and cefuroxime (39.26\%) [Table 4]. Among the three fluoroquinolones, the MIC of levofloxacin is minimum in all respiratory isolates [Table 5].

\section{Discussion}

The study results indicated that Klebsiella pneumoniae (38.14\%) was the most predominant pathogen

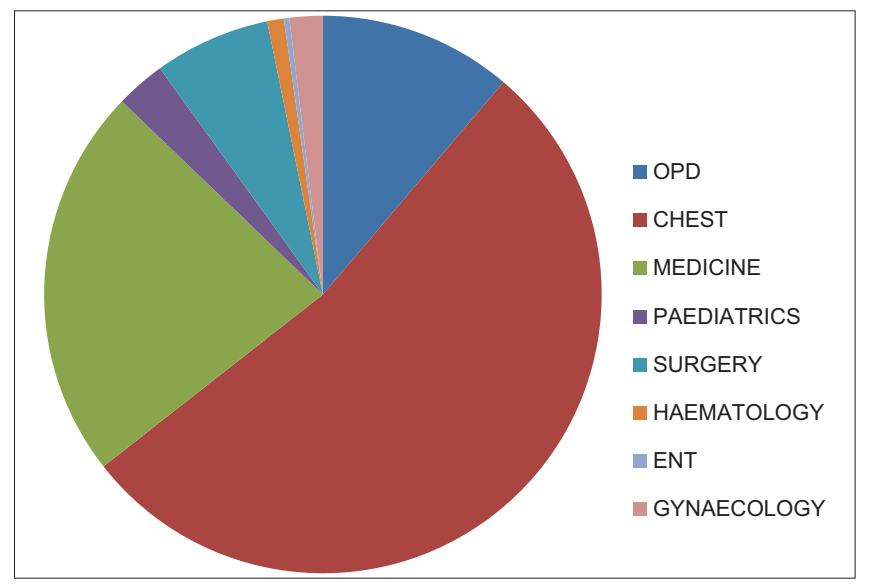

Figure 1: Wardwise distribution of the pathogen

in LRTIs same as observed in a study from South India ${ }^{[10]}$ and in Nigeria, ${ }^{[11]}$ who also reported that $H$. influenzae was the second most common pathogen in LRTIs. This was in contrast to the current study, which revealed Moraxella spp. (16.02\%) as the next common bacterium isolated. The predominance of Gram-negative bacteria (76.6\%) among the LRTIs in this study was also observed by a recent study from Nepal by Mishra et al. who reported 84.1\% occurrence. ${ }^{[12]}$

In this study, LRTI was more common in males than females, which were consistent with other studies from India. ${ }^{[13]}$ This was due to more prevalence of associated risk factors (e.g., smoking, chronic alcoholism, COPD) of pneumonia in Indian males than females. ${ }^{[14]}$

The maximum number of patients were from older age group, $45-60$ years $(29.80 \%)$ and $>60$ years $(25 \%)$. This was in accordance with a study from India ${ }^{[15]}$ and Finland where the rate of pneumonia increased for each year of age over 50 years. ${ }^{[16]}$

Management of LRTIs had been a challenge to the physicians, more recently due to the emergence of multi-drug 


\begin{tabular}{lccc}
\hline Table 5: MIC ( $\boldsymbol{\mu g} / \mathbf{m l})$ of three fluroquinolones against commonly isolated pathogens of LRTI & \\
\hline Organisms & MIC $_{90}$ ciprofloxacin & MIC $_{90}$ ofloxacin & MIC $_{90}$ levofloxacin \\
Klebsiella spp. (119) & 8 & 8 & 1 \\
Moraxella spp. (50) & 0.12 & 0.12 & 0.06 \\
Staphylococcus aureus (29) & 1 & 2 & 0.5 \\
Pneumococcus (44) & 2 & 2 & 1 \\
Pseudomonas spp. (31) & 4 & 8 & 2 \\
Citrobacter spp. (6) & 4 & 4 & 4 \\
Escherichia coli (14) & 8 & 8 & 4 \\
Acinetobacter spp. (12) & 4 & 4 & 2 \\
Haemophilus influenzae (7) & 0.5 & 0.03 & 0.03 \\
\hline
\end{tabular}

MIC: Minimum inhibitory concentration, LRTI: Lower respiratory tract infections

resistance. ${ }^{[17]}$ Although the strains of $S$. pneumoniae in this study had shown high degree of resistance to cotrimoxazole (63.6\%), ciprofloxacin (36.4\%), ofloxacin (25\%) and ampicillin (22.7\%), sensitivity to levofloxacin, erythromycin, amoxicillin-clavulanic acid, cefixime, cefuroxime, cefotaxime was 86-96\%. K. pneumoniae, E. coli, Acinetobacter spp., Citrobacter spp. and $S$. aureus were $80-90 \%$ resistant to amoxicillin, $65-85 \%$ resistant to amoxicillin-clavulanic acid, 60-92\% resistant to cefuroxime, $55-72 \%$ resistant to cefotaxime, $30-68 \%$ resistant to doxycycline. Cefoperazone-sulbactam was 50-65\% resistant in E. coli, Acinetobacter spp., Citrobacter spp., S. aureus but only $26 \%$ resistant in Klebsiella. Cefixime was $70-86 \%$ resistant in K. pneumoniae, E. coli, Acinetobacter spp. and Citrobacter spp. It was found that $S$. aureus and Klebsiella were $70-85 \%$ resistant to macrolides. Levofloxacin was most sensitive (83.33\%) and cotrimoxazole was most resistant $(78.29 \%)$ in all isolates.

In German CAPNETZ surveillance study, 163 pneumococcal isolates from 556 patients with pneumococcal pneumonia were analyzed for fluoroquinolone resistance and none exhibited fluoroquinolone resistance. ${ }^{[15]}$ In one study $S$. pneumoniae was $100 \%$ sensitive to levofloxacin; no resistance had been detected. However, it was resistant to erythromycin, clindamycin and cotrimoxazole. ${ }^{[18]}$ In a study from Greece, among 588 bacteria isolated during the period 1/2009-12/2012 from patients with community-acquired respiratory tract infections, only two were resistant to newer fluoroquinolones. ${ }^{[19]}$ A multivariate analytical study including 981 patients with pneumococcal pneumonia, 46 (4.7\%) had levofloxacin-nonsusceptible $S$. pneumoniae, of which 23 were classified as healthcare-associated infection. ${ }^{[20]}$

In a recent study on LRTIs, Panda et al. from Andhra Pradesh reported $K$. pneumoniae (31.3\%) as the most frequent causative agent followed by $S$. aureus (26.5\%). The same study had reported that fluoroquinolones showed better in vitro sensitivities when compared with cephalosporins indicating the prevalence of ESBL's in Gram-negative bacteria. ${ }^{[21]}$

The susceptibility pattern in this study revealed that cotrimoxazole, amoxicillin, amoxicillin-clavulanic acid, cefuroxime and erythromycin were not so active against any bacteria. This indicated that resistance had evolved due to long-term use of these quite affordable drugs in the community. ${ }^{[11]}$ Among all the first line drugs against LRTIs levofloxacin showed highest sensitivity in vitro. In vivo levofloxacin had a favorable pharmacokinetic profile in plasma of patients with severe LRTIs. ${ }^{[22]}$ After a single oral dose of levofloxacin, the drug is rapidly distributed in the bronchopulmonary tissues achieving its peak concentration in plasma in $1 \mathrm{~h}$ and epithelial lining cells in $1 \frac{1 / 2}{h} \cdot{ }^{[23]}$ In accordance to a study done in Kerala, this study also proposed levofloxacin as an effective oral antibacterial agent for LRTI patients. $^{[24]}$

The strength of this study was that it had dealt with a large number of cases including both community and hospital acquired infections, thereby being able to distinguish between the common pathogens and their antimicrobial resistance pattern of CAP and HAP.

The study had its own limitation in that the causative pathogens of atypical pneumonia could not be identified as we used routine culture media for isolation.

\section{Conclusion}

Considering both community and hospital acquired LRTIs, the most common organism isolated was K. pneumoniae (38.14\%). The occurrence of multidrug resistance even at the community level should be considered as a matter of concern. In this study it was found that the overall susceptibility pattern was $<50 \%$ for amoxicillin, amoxicillin-clavulanic acid, cefuroxime, cotrimoxazole and erythromycin whereas for cefotaxime, cefixime, and cefoperazone-sulbactum it was 60.08\%, 51.59\%, 69.04\% respectively. The susceptibility to ciprofloxacin, ofloxacin and levofloxacin was $66.67 \%, 70.19 \%$ and $83.33 \%$, respectively. Therefore, it was found that levofloxacin, (being an oral drug with good compliance) had good activity against respiratory pathogens and could be used for empiric treatment in LRTI.

\section{References}

1. Sharma BB, Singh V. Indian pneumonia guidelines. Lung India 2012;29:307-8.

2. Chang AB, Chang CC, O'Grady K, Torzillo PJ. Lower respiratory tract infections. Pediatr Clin North Am 2009;56:1303-21. 
3. Boloursaz MR, Lotfian F, Aghahosseini F, Cheraghvandi A, Khalilzadeh S, Farjah A, et al. Epidemiology of lower respiratory tract infections in children. J Compr Pediatr 2013;3:93-8.

4. Guthrie R. Community-acquired lower respiratory tract infections: Etiology and treatment. Chest 2001;120:2021-34.

5. Hooper D. Quinolones. In: Mandell GL, Bennett JE, Dolin R, editors. Mandell, Douglas, and Bennett's Principles and Practice of Infectious Diseases. $5^{\text {th }}$ ed. Philadelphia: Churchill Livingstone; 2000. p. 404-23.

6. Turnidge J. Pharmacokinetics and pharmacodynamics of fluoroquinolones. Drugs 1999;58 Suppl 2:29-36.

7. Walker RC. The fluoroquinolones. Mayo Clin Proc 1999;74:1030-7.

8. Sarkar S, Sen Gupta M, Saha P, Nag S. To compare the in vitro activity of 3 popular fluoroquinolones against common respiratory and middle ear pathogens. Natl J Otorhinolaryngol Head Neck Surg 2013;3:21-3.

9. Murray PR, Washington JA. Microscopic and baceriologic analysis of expectorated sputum. Mayo Clin Proc 1975;50:339-44.

10. Ramana KV, Kalaskar A, Rao M, Rao SD. Aetiology and antimicrobial susceptibility patterns of lower respiratory tract infections (LRTI) in a rural Tertiary Care Teaching Hospital in Karimnagar, South India. Am J Infect Dis Microbiol 2013;1:101-5.

11. Egbe CA, Ndiokwere C, Omoregie R. Microbiology of lower respiratory tract infections in Benin City, Nigeria. Malays J Med Sci 2011;18:27-31

12. Mishra SK, Kattel HP, Acharya J. Recent trend of bacterial aetiology of lower respiratory tract infections in a Tertiary Care Centre of Nepal. Int J Infect Microbiol 2012;1:3-8.

13. Oberoi A, Aggrawal A. Bacteriological profile, serology and antibiotic sensitivity pattern of microorganisms from community acquired pneumonia. JK Sci 2006;8:79-82.

14. Bansal S, Kashyap S, Pal LS, Goel A. Clinical and bacteriological profile of community acquired pneumonia in Shimla, Himachal Pradesh. Indian J Chest Dis Allied Sci 2004;46:17-22.

15. Pletz MW, van der Linden M, von Baum H, Duesberg CB, Klugman KP, Welte T. Low prevalence of fluoroquinolone resistant strains and resistance precursor strains in Streptococcus pneumoniae from patients with community-acquired pneumonia despite high fluoroquinolone usage. Int J Med Microbiol 2011;1:53-7.

16. Bartlett JG, Dowell SF, Mandell LA, File Jr TM, Musher DM, Fine MJ.
Practice guidelines for the management of community-acquired pneumonia in adults. Infectious Diseases Society of America. Clin Infect Dis 2000;31:347-82.

17. Whaley LE, Businger AC, Dempsey PP, Linder JA. Visit complexity, diagnostic uncertainty, and antibiotic prescribing for acute cough in primary care: A retrospective study. BMC Fam Pract 2013;14:120.

18. Altinkanat Gelmez G, Soysal A, Kuzdan C, Karadag B, Hasdemir U, Bakir M, et al. Serotype distribution and antibiotic susceptibilities of Streptococcus pneumoniae causing acute exacerbations and pneumonia in children with chronic respiratory diseases. Mikrobiyol Bul 2013;47:684-92.

19. Maraki S, Papadakis SL. Antimicrobial resistance trends amongcommunity-acquired respiratory tract pathogens in Greece, 2009-2012. The Scientific WorldJ2014;2014:doi:10.1155/2014/941564

20. Kang CI, Song JH, Kim SH, Chung DR, Peck KR, So TM, et al. Risk factors for levofloxacin-nonsusceptible Streptococcus pneumoniae in community-acquired pneumococcal pneumonia: A nested case-control study. Eur J Clin Microbiol Infect Dis 2014;33:55-9.

21. Panda S, Nandini BP, Ramani TV. Lower respiratory tract infections-bacteriological profile and antibiogram pattern. Int J Curr Res Rev 2012;4:149-55.

22. Kontou P, Manika K, Chatzika K, Papaioannou M, Sionidou M, Pitsiou G, et al. Pharmacokinetics of moxifloxacin and high-dose levofloxacin in severe lower respiratory tract infections. Int J Antimicrob Agents 2013;42:262-7.

23. Zhang J, Xie X, Zhou X, Chen YQ, Yu JC, Cao GY, et al. Permeability and concentration of levofloxacin in epithelial lining fluid in patients with lower respiratory tract infections. J Clin Pharmacol 2010;50:922-8.

24. Ahmed SM, Jakribettu RP, Meletath SK, Arya B, Shakir VPA. Lower respiratory tract infections (LTRIs): An insight into the prevalence and the antibiogram of the gram negative, respiratory, bacterial agents. J Clin Diagn Res 2013;7:253-6.

How to cite this article: Sarkar S, Chakraborty A, Sengupta M, Ghosh S, Mukhopadhyay S, SenGupta M. In vitro activity of levofloxacin against lower respiratory tract pathogens. J Basic Clin Pharma 2015;6:89-93.

Source of Support: Nil, Conflict of Interest: None declared. 